\title{
Common Fixed Point Results for Fuzzy Mappings on Complex-Valued Metric Spaces with Homotopy Results
}

\author{
Humaira ${ }^{1}$, Muhammad Sarwar ${ }^{1, * \mathbb{D}}$ and Poom Kumam ${ }^{2,3,4, * \text { (D) }}$ \\ 1 Department of Mathematics, University of Malakand, Dir(L), Khyber Pakhtunkhwa 18800, Pakistan; \\ humaira.swatpk@gmail.com \\ 2 KMUTTFixed Point Research Laboratory, Department of Mathematics, Room SCL 802 Fixed Point \\ Laboratory, Science Laboratory Building, Faculty of Science, King Mongkut's University of Technology \\ Thonburi (KMUTT), 126 Pracha-Uthit Road, Bang Mod, Thrung Khru, Bangkok 10140, Thailand \\ 3 KMUTT-Fixed Point Theory and Applications Research Group (KMUTT-FPTA), Theoretical and \\ Computational Science Center (TaCS), Science Laboratory Building, Faculty of Science, \\ King Mongkut's University of Technology Thonburi (KMUTT), 126 Pracha-Uthit Road, Bang Mod, \\ Thrung Khru, Bangkok 10140, Thailand \\ 4 Department of Medical Research, China Medical University Hospital, China Medical University, \\ Taichung 40402, Taiwan \\ * Correspondence: sarwarswati@gmail.com (M.S.); poom.kum@kmutt.ac.th (P.K.)
}

Received: 6 November 2018; Accepted: 22 December 2018; Published: 8 January 2019

\begin{abstract}
Owing to the notion of a complex-valued metric space, we prove fixed point results, which generalize some common fixed point results under contractive condition for rational expression in the context of complex-valued metric spaces. In application, we present a homotopy result to apply the results obtained herein.
\end{abstract}

Keywords: complex valued metric space; fuzzy mappings; fixed point; common fixed point; cauchy sequence; contractive condition

MSC: 26A33; 34A08; 35B40

\section{Introduction and Preliminaries}

Fixed point theory has shown the importance of theoretical subjects, which are directly applicable in different applied fields of science. In particular, it plays an important role in the investigation of existence of solutions to differential and integral equations, which direct the behaviour of several real life problems for which the existence of solution is critical. In 1922, Banach provided a general iterative method to construct a fixed point result and proved its uniqueness under linear contraction in complete metric spaces [1]. Researchers solved various types of concrete problems with the help of Banach contraction principal (for instance, we refer the readers to [2-7]).

Nadler [8] generalized the Banach contraction technique to multivalued mappings, which are further extended by researchers in the recent years (see [9,10]). Heilpern [11] introduced the concept of fuzzy mappings to extend Banach fixed point technique in the setting of metric linear spaces. Many researchers extended the work of Heilpern and obtained fuzzy fixed point results (for details, we refer to [12-21]). Dass and Gupta [22] utilized the Banach's fixed point technique for rational contraction in metric spaces, which is further extended to different spaces by several researchers. Meanwhile, researchers realized that, due to vectors' decision, rational contraction is not meaningful in cone metric spaces. 
Recently, Azam et al. [23] established a special class of cone metric space where they created the possibility to utilize rational type contraction for vector division in the form of complex numbers. The newly established class is known as a complex-valued metric space where they obtained common fixed point results for rational contraction. Subsequently, Sintunavarat et al. [24,25], Klien-eam et al. [26,27], Rozkard et al. [28], Sitthikul et al. [29] and Kutbi et al. [30] derived results of common fixed points satisfying different types of rational contraction in complex-valued metric spaces.

In the recent years, Samet et al. [31] initiated the concept of $\alpha$-admissible mappings. They proved common fixed point results for such type of mappings. Asl [32] and Kutbi et al. [33] further improved the notion of $\alpha$-admissible mappings by introducing coupled $\alpha$-admissible mappings and $\alpha^{*}-\psi$-admissible mappings and obtained fixed point results for self mappings and multivalued mappings, respectively.

Motivated by the above-mentioned work, in this paper, we study common fixed point for fuzzy mappings by adopting the concept of coupled $\alpha^{*}$-admissible mapping in complex-valued metric spaces. Our work generalizes the results of [34] for fuzzy mappings.

We organize our paper as, in Section 1, we have provided some basic definitions and lemmas upon which our results are based. In Section 2, we obtained our main results. As an application, we derived common fixed point results for multivalued mappings, which generalize many results already proved in literature. Also in this section, we have constructed an appropriate example to show the validity of our main results. In Section 3, we have provided an application of one of our results by proving a homotopy result. In Section 4 , we have concluded our results.

Definition 1 ([23]). Assume $C$ is the set of complex numbers. For $\varepsilon_{1}, \varepsilon_{2} \in C$, we define a partial order $\precsim$ on $C$ as follows:

$$
\varepsilon_{1} \precsim \varepsilon_{2} \text { iff } \operatorname{Re}\left(\varepsilon_{1}\right) \leq \operatorname{Re}\left(\varepsilon_{2}\right) \text {, and } \operatorname{Im}\left(\varepsilon_{1}\right) \leq \operatorname{Im}\left(\varepsilon_{2}\right) \text {. }
$$

It follows that

$$
\varepsilon_{1} \precsim \varepsilon_{2}
$$

if one of the following conditions is satisfied:

(Ci) $\operatorname{Re}\left(\varepsilon_{1}\right)<\operatorname{Re}\left(\varepsilon_{2}\right), \operatorname{Im}\left(\varepsilon_{1}\right)=\operatorname{Im}\left(\varepsilon_{2}\right)$,

(Cii) $\operatorname{Re}\left(\varepsilon_{1}\right)=\operatorname{Re}\left(\varepsilon_{2}\right), \operatorname{Im}\left(\varepsilon_{1}\right)<\operatorname{Im}\left(\varepsilon_{2}\right)$,

(Ciii) $\operatorname{Re}\left(\varepsilon_{1}\right)<\operatorname{Re}\left(\varepsilon_{2}\right), \operatorname{Im}\left(\varepsilon_{1}\right)<\operatorname{Im}\left(\varepsilon_{2}\right)$,

(Civ) $\operatorname{Re}\left(\varepsilon_{1}\right)=\operatorname{Re}\left(\varepsilon_{2}\right), \operatorname{Im}\left(\varepsilon_{1}\right)=\operatorname{Im}\left(\varepsilon_{2}\right)$.

In particular, we write $\varepsilon_{1} \precsim \varepsilon_{2}$ if $\varepsilon_{1} \neq \varepsilon_{2}$ and one of (Ci), (Cii) and (Ciii) is satisfied, and $\varepsilon_{1}=\varepsilon_{2}$ if and only if (Civ) is satisfied. Note that

(i) $0 \precsim \varepsilon_{1} \precsim \varepsilon_{2} \Rightarrow\left|\varepsilon_{1}\right|<\left|\varepsilon_{2}\right|, \forall \varepsilon_{1}, \varepsilon_{2} \in C$,

(ii) $\varepsilon_{1} \precsim \varepsilon_{2}$ and $\varepsilon_{2} \prec \varepsilon_{3} \Rightarrow \varepsilon_{1} \prec \varepsilon_{3}, \forall \varepsilon_{1}, \varepsilon_{2}, \varepsilon_{3} \in C$.

Definition 2 ([23]). Let $\mathcal{S}$ be a nonempty set and $\sigma: \mathcal{S} \times \mathcal{S} \rightarrow$ C be a mapping satisfying the following conditions:

(1) $0 \precsim \sigma(\mathrm{z}, w)$, for all $\mathrm{z}, w \in \mathcal{S}$ and $\sigma(\mathrm{z}, w)=0$ if and only if $\mathrm{z}=w$;

(2) $\sigma(\mathrm{z}, w)=\sigma(w, \mathrm{z})$, for all $\mathrm{z}, w \in \mathcal{S}$;

(3) $\sigma(\mathrm{z}, w) \precsim \sigma\left(\mathrm{z}, \mathrm{z}_{1}\right)+\sigma\left(\mathrm{z}_{1}, w\right)$, for all $\mathrm{z}, \mathrm{z}_{1}, w \in \mathcal{S}$.

Then, $(\mathcal{S}, \sigma)$ is called a complex-valued metric space.

Definition 3 ([23]). A point $\mathrm{z} \in \mathcal{S}$ is known as an interior point of a set $\mathrm{Z} \subseteq \mathcal{S}$, if we find $0 \prec \epsilon \in C$ such that,

$$
\mathfrak{B}(\mathrm{z}, \epsilon)=\{\mathrm{w} \in \mathcal{S}: \sigma(\mathrm{z}, \mathrm{w}) \prec \epsilon\} \subseteq \mathrm{Z} .
$$

A point $\mathrm{z} \in \mathrm{Z}$ is known as the limit point of $\mathrm{Z}$, if there exists an open ball $\mathfrak{B}(\mathrm{z}, \epsilon)$ such that

$$
\mathfrak{B}(\mathrm{z}, \epsilon) \cap(Z \backslash\{\mathrm{z}\}) \neq \phi,
$$


where $0 \prec \epsilon \in C$. A subset $Z$ of $\mathcal{S}$ is said to be open if each point of $Z$ is an interior point of $Z$. Furthermore, $\mathrm{Z}$ is said to be closed if it contains all its limit points.

The family

$$
\mathcal{B}=\{\mathfrak{B}(\mathrm{z}, \epsilon): \mathrm{z} \in \mathcal{S}, 0 \prec \epsilon\}
$$

is a sub-basis for a Hausdorff topology $\mathfrak{T}$ on $\mathcal{S}$.

Now, recall some definitions from $[9,26]$.

Let $(\mathcal{S}, \sigma)$ be a complex-valued metric space. Throughout this paper, we have denoted the family of all nonempty closed bounded subsets of complex-valued metric space $\mathcal{S}$ by $\mathcal{C B}(\mathcal{S})$. For $v \in C$, we denote

$$
s(v)=\{\mathrm{z} \in C: v \preceq \mathrm{z}\}
$$

and, for $\mathrm{w} \in \mathcal{S}$ and $B \in \mathcal{C B}(\mathcal{S})$,

$$
s(\mathrm{w}, B)=\cup_{b \in B} s(\sigma(\mathrm{w}, b))=\cup_{b \in B}\{z \in C: \sigma(\mathrm{w}, b) \preceq \mathrm{z}\} .
$$

For $\mathrm{A}, B \in \mathcal{C} \mathcal{B}(\mathcal{S})$, we denote

$$
s(A, B)=\left(\cap_{p \in A} s(p, B)\right) \cap\left(\cap_{q \in B} s(q, A)\right) .
$$

Let $\tau$ be a multivalued mapping from $\mathcal{S}$ into $\mathcal{C B}(\mathcal{S})$. For $z \in \mathcal{S}$ and $Q \in \mathcal{C B}(\mathcal{S})$, we define

$$
\mathcal{W}_{z}(Q)=\{\sigma(z, q): q \in Q\} .
$$

Thus, for $z, w \in \mathcal{S}$,

$$
\mathcal{W}_{z}(\tau w)=\{\sigma(z, u): u \in \tau w\} .
$$

Lemma 1 ([5]). Let $(\mathcal{S}, \sigma)$ be complex-valued metric space:

(i) Let $z, w \in C$. If $z \preceq w$, then $s(z) \subset s(w)$.

(ii) Let $z \in \mathcal{S}$ and $D \in \mathbb{N}(\mathcal{S})$. If $\delta \in s(z, D)$, then $z \in D$.

(iii) Let $w \in C, P, Q \in \mathcal{C B}(\mathcal{S})$ and $p \in P$. If $v \in s(P, Q)$, then $z \in s(p, Q)$ for all $p \in P$ or $z \in s(P, q)$ for all $q \in Q$.

Definition 4 ([23]). Let $\left\{w_{r}\right\}$ be a sequence in complex-valued metric space $(\mathcal{S}, \sigma)$ and $w \in \mathcal{S}$; then,

(i) $w$ is said to be a limit point of $\left\{w_{r}\right\}$ if for each $0 \prec \epsilon \in C$ there exists an $r_{0} \in N$ such that $\sigma\left(w_{r}, w\right) \preceq \epsilon$ for all $r \succeq r_{0}$ and written as $\lim _{r \rightarrow \infty} w_{r}=w$.

(ii) $\left\{w_{r}\right\}$ is a Cauchy sequence if for any $0 \prec \epsilon \in C$ there exists an $r_{0} \in N$ such that $\sigma\left(w_{r}, w_{r+t}\right) \prec \epsilon$ for all $r \succ r_{0}$ where $t \in N$.

(iii) We say that $(\mathcal{S}, \sigma)$ is complete complex-valued metric space if every Cauchy sequence in $\mathcal{S}$ converges to a point in $\mathcal{S}$.

Definition 5 ([12]). Let $(V, \sigma)$ be a metric space. A fuzzy set $\mathcal{A}$ is characterized by its membership function $f_{\mathcal{A}}: V \rightarrow[0,1]$, which assigns a grade of membership to each element of $\mathcal{A}$ between 0 and 1 . For simplicity, we denote $f_{\mathcal{A}}(u)$ by $\mathcal{A}(u)$. The $\alpha$-level set of a fuzzy set $\mathcal{A}$ is denoted here by $[\mathcal{A}]_{\alpha}$ which is defined as follows:

$$
\begin{gathered}
{[\mathcal{A}]_{\alpha}=\{u: \mathcal{A}(u) \geq \alpha\} \text { if } \alpha \in(0,1],} \\
{[\mathcal{A}]_{0}=\{\overline{u: \mathcal{A}(u)>0\}} .}
\end{gathered}
$$

Here, $\overline{\mathcal{A}}$ denotes the closer of the set $A$. 
Definition 6 ([12]). Let $\mathfrak{L}(\mathcal{S})$ be the family of all fuzzy sets in a metric space $\mathcal{S}$. For $G, H \in \mathfrak{L}(\mathcal{S}), G \subset H$ means $G(z) \leq H(z)$ for each $z \in \mathcal{S}$.

Definition 7 ([11]). Suppose $\mathcal{S}$ is an arbitrary set, and $\mathcal{Y}$ is a metric space. If $G: \mathcal{S} \rightarrow \mathfrak{L}(\mathcal{Y})$, then $G$ is said to be a fuzzy mapping. A fuzzy mapping $G$ is a fuzzy subset on $\mathcal{S} \times \mathcal{Y}$ with a membership function $G(w)(z)$. The function $G(w)(z)$ is the grade of membership of $z$ in $G(w)$.

Definition 8 ([20]). Assume that $(\mathcal{S}, \sigma)$ is a complex-valued metric space and $\mathcal{G}_{1}, \mathcal{G}_{2}: \mathcal{S} \rightarrow \mathfrak{L}(\mathcal{S})$ are fuzzy mappings. A point $w \in \mathcal{S}$ is said to be a fuzzy fixed point of $\mathcal{G}_{1}$ if $w \in\left[\mathcal{G}_{1} w\right]_{\alpha}$ for some $\alpha \in[0,1]$ and $w$ is said to be a common fuzzy fixed point of $\mathcal{G}_{1}, \mathcal{G}_{2}$ if $w \in\left[\mathcal{G}_{1} w\right]_{\alpha} \cap\left[\mathcal{G}_{2} w\right]_{\alpha}$. If $\alpha=1$, then $w$ is known as a common fixed point of fuzzy mappings.

Definition 9 ([20]). Let $(\mathcal{S}, \sigma)$ be a complex valued metric space. A multivalued mapping $\mathcal{G}: \mathcal{S} \rightarrow 2^{C}$ is said to be bounded from below if, for each $w \in \mathcal{S}$, there exists $z_{w} \in C$ such that

$$
z_{w} \preceq u
$$

for all $u \in \mathcal{G} w$.

Definition 10 ([20]). Let $(\mathcal{S}, \sigma)$ be complex valued metric space. The fuzzy mapping $\mathcal{G}: \mathcal{S} \rightarrow \mathfrak{L}(\mathcal{S})$ is said to have greatest lower bound property $(\mathrm{glb})$ on $(\mathcal{S}, \sigma)$ if, for any $w \in \mathcal{S}$ associated with some $\alpha$, the multivalued mapping $F_{z}: \mathcal{S} \rightarrow 2^{C}$ defined by

$$
F_{z}(w)=\mathrm{W}_{z}([\mathcal{G} w])
$$

is bounded from below that is, for any $z, w \in \mathcal{S}$, there exists an element $I_{z}\left([\mathcal{S} w]_{\alpha} \in \mathcal{S}\right)$ such that $I_{z}\left([\mathcal{S} w]_{\alpha} \preceq u\right.$, for all $u \in \mathrm{W}_{z}([\mathcal{G} w])$, where $I_{z}\left([\mathcal{S} w]_{\alpha}\right.$ is a lower bound of $\mathcal{G}$ associated with some $(z, w)$.

Definition 11 ([20]). Let $(\mathcal{S}, \sigma)$ be complex-valued metric space and the fuzzy mapping $\mathcal{G}_{1}: \mathcal{S} \rightarrow \mathfrak{L}(\mathcal{S})$ satisfies the greatest lower bound property (glb property) on $(\mathcal{S}, \sigma)$. Then, for any $w \in \mathcal{S}$ and $\alpha \in(0,1]$, the greatest lower bound of $\mathrm{W}_{w}\left(\left[\mathcal{G}_{1} y\right]_{\alpha}\right)$ exists in $C$ for all $w, y \in \mathcal{S}$. Here, we have denoted $\sigma\left(w,\left[\mathcal{G}_{1} y\right]_{\alpha}\right)$ by the glb of $\mathrm{W}_{w}\left(\left[\mathcal{G}_{1} y\right]_{\alpha}\right)$, i.e.,

$$
\sigma\left(w,\left[\mathcal{G}_{1} y\right]_{\alpha}\right)=\inf \left\{\sigma(w, u): u \in\left[\mathcal{G}_{1} y\right]_{\alpha}\right\} .
$$

Remark 1 ([26]). Let $(\mathcal{S}, \sigma)$ be a complex-valued metric space. If $C=\mathbb{R}$, then $(\mathcal{S}, \sigma)$ is a metric space. Furthermore, $\mathrm{H}(A, B)=\inf s(A, B)$ is the Hausdorff distance induced by $\sigma$, where $A, B \in \mathcal{C B}(\mathcal{S})$.

Definition 12 ([34]). Let $P, Q: \mathcal{S} \rightarrow \mathcal{C B}(\mathcal{S})$, and let $\varrho: \mathcal{S} \times \mathcal{S} \rightarrow[0,+\infty)$. Then, we say that $P, Q$ are coupled $\varrho^{*}$-admissible if $\varrho(u, v) \geq 1$ implies $\varrho^{*}(P u, Q v) \geq 1$ for all $u, n \in \mathcal{S}$, where $\varrho^{*}(P u, Q v)=$ $\inf \{\varrho(u, v): u \in P u, v \in Q v\}$.

\section{Main Results}

Theorem 1. Let $(\mathcal{S}, \sigma)$ be complete complex valued metric space and $P, Q: \mathcal{S} \rightarrow \mathfrak{L}(\mathcal{S})$ be coupled $\varrho^{*}$-admissible mappings, which satisfy the glb property. Assume that, for $P$ and $Q$, the following condition holds

$$
\begin{aligned}
& \xi \sigma(u, v)+\frac{\zeta \sigma\left(u,[P u]_{\alpha}\right) \sigma\left(v,[Q v]_{\alpha}\right)+\eta \sigma\left(v,[P u]_{\alpha}\right) \sigma\left(u,[Q v]_{\alpha}\right)}{1+\sigma(u, v)} \\
+ & \frac{\rho \sigma\left(u,[P u]_{\alpha}\right) \sigma\left(u,[Q v]_{\alpha}\right)+\delta \sigma\left(v,[P u]_{\alpha}\right) \sigma\left(v,[Q v]_{\alpha}\right)}{1+\sigma(u, v)} \\
\epsilon & Q^{*}\left([P u]_{\alpha},[Q v]_{\alpha}\right) s\left([P u]_{\alpha},[Q v]_{\alpha}\right)
\end{aligned}
$$


for all $v, u \in \bar{B}\left(u_{0}, t\right)$, where $u_{0} \in \mathcal{S}$ and $0 \prec t \in C$, with

$$
(1-\mathfrak{Z}) t \in s\left(u_{0},\left[P u_{0}\right]_{\alpha}\right),
$$

where $\zeta, \eta$ and $\xi$ are nonnegative real numbers such that $\mathfrak{Z}=\frac{\xi+\rho}{1-\zeta-\rho}<1$. Suppose $\varrho\left(u_{0}, u_{1}\right) \geq 1$, for some $u_{1} \in \bar{B}\left(u_{0}, t\right)$ and for each $v, u \in \mathcal{S}$. Let $[P u]_{\alpha},[Q v]_{\alpha}$ be nonempty closed, bounded subsets of $\mathcal{S}$ associated with some $\alpha \in(0,1]$. If $\left\{u_{q}\right\}$ is a sequence in $\bar{B}\left(u_{0}, t\right)$ with $\varrho\left(u_{q}, u_{q+1}\right) \geq 1$ and $u_{q} \rightarrow z$ as $q \rightarrow+\infty$, then $\varrho\left(u_{q}, z\right) \geq 1$ for all $q$.

Then, there exists a point $u^{*} \in \bar{B}\left(u_{0}, t\right)$ such that $u^{*} \in\left[P u^{*}\right]_{\alpha} \cap\left[Q u^{*}\right]_{\alpha}$.

Proof. Take arbitrary point $u_{0} \in \mathcal{S}$ since

$$
(1-\mathfrak{Z}) t \in s\left(u_{0},\left[P u_{0}\right]_{\alpha}\right) \quad \Rightarrow(1-\mathfrak{Z}) t \in \bigcup_{u_{1} \in\left[P u_{0}\right]_{\alpha}} s\left(\sigma\left(u_{0}, u_{1}\right)\right) .
$$

Since $\left[P u_{0}\right]_{\alpha}$ is a nonempty, closed and bounded; therefore, for some $u_{1} \in\left[P u_{0}\right]_{\alpha}$, one can write

$$
(1-\mathfrak{Z}) t \in s\left(\sigma\left(u_{0}, u_{1}\right)\right)
$$

By definition, we obtain

$$
\left.\sigma\left(u_{0}, u_{1}\right)\right) \preceq(1-\mathfrak{Z}) t
$$

which yields

$$
\left.\mid \sigma\left(u_{0}, u_{1}\right)\right)|\leq(1-\mathfrak{Z})| t \mid .
$$

Hence, $u_{1} \in \bar{B}\left(u_{0}, t\right)$. As we have supposed that $\varrho\left(u_{0}, u_{1}\right) \geq 1$, and $\{P, Q\}$ is coupled $\varrho^{*}$-admissible, thus $\varrho^{*}\left(\left[P u_{0}\right]_{\alpha},\left[Q u_{1}\right]_{\alpha}\right)>1$. Using (2), we have

$$
\begin{aligned}
& \zeta \sigma\left(u_{0}, u_{1}\right)+\frac{\zeta \sigma\left(u_{0},\left[P u_{0}\right]_{\alpha}\right) \sigma\left(u_{1},\left[Q u_{1}\right]_{\alpha}\right)+\eta \sigma\left(u_{1},\left[P u_{0}\right]_{\alpha}\right) \sigma\left(u_{0},\left[Q u_{1}\right]_{\alpha}\right)}{1+\sigma\left(u_{0}, u_{1}\right)} \\
+ & \frac{\rho \sigma\left(u_{0},\left[P u_{0}\right]_{\alpha}\right) \sigma\left(u_{0},\left[Q u_{1}\right]_{\alpha}\right)+\delta \sigma\left(u_{1},\left[P u_{0}\right]_{\alpha}\right) \sigma\left(u_{1},\left[Q u_{1}\right]_{\alpha}\right)}{1+\sigma\left(u_{0}, u_{1}\right)} \\
\epsilon & \varrho^{*}\left(\left[P u_{0}\right]_{\alpha},\left[Q u_{1}\right]_{\alpha}\right) s\left(\left[P u_{0}\right]_{\alpha},\left[Q u_{1}\right]_{\alpha}\right) .
\end{aligned}
$$

Using Lemma 1 (iii), we obtain

$$
\begin{aligned}
& \xi \sigma\left(u_{0}, u_{1}\right)+\frac{\zeta \sigma\left(u_{0},\left[P u_{0}\right]_{\alpha}\right) \sigma\left(u_{1},\left[Q u_{1}\right]_{\alpha}\right)+\eta \sigma\left(u_{1},\left[P u_{0}\right]_{\alpha}\right) \sigma\left(u_{0},\left[Q u_{1}\right]_{\alpha}\right)}{1+\sigma\left(u_{0}, u_{1}\right)} \\
+ & \frac{\rho \sigma\left(u_{0},\left[P u_{0}\right]_{\alpha}\right) \sigma\left(u_{0},\left[Q u_{1}\right]_{\alpha}\right)+\delta \sigma\left(u_{1},\left[P u_{0}\right]_{\alpha}\right) \sigma\left(u_{1},\left[Q u_{1}\right]_{\alpha}\right)}{1+\sigma\left(u_{0}, u_{1}\right)} \\
\epsilon & Q^{*}\left(\left[P u_{0}\right]_{\alpha},\left[Q u_{1}\right]_{\alpha}\right) s\left(u_{1},\left[Q u_{1}\right]_{\alpha}\right) .
\end{aligned}
$$

By definition, there is some $u_{2} \in\left[Q u_{1}\right]_{\alpha}$ such that

$$
\begin{aligned}
& \xi \sigma\left(u_{0}, u_{1}\right)+\frac{\zeta \sigma\left(u_{0},\left[P u_{0}\right]_{\alpha}\right) \sigma\left(u_{1},\left[Q u_{1}\right]_{\alpha}\right)+\eta \sigma\left(u_{1},\left[P u_{0}\right]_{\alpha}\right) \sigma\left(u_{0},\left[Q u_{1}\right]_{\alpha}\right)}{1+\sigma\left(u_{0}, u_{1}\right)} \\
+ & \frac{\rho \sigma\left(u_{0},\left[P u_{0}\right]_{\alpha}\right) \sigma\left(u_{0},\left[Q u_{1}\right]_{\alpha}\right)+\delta \sigma\left(u_{1},\left[P u_{0}\right]_{\alpha}\right) \sigma\left(u_{1},\left[Q u_{1}\right]_{\alpha}\right)}{1+\sigma\left(u_{0}, u_{1}\right)} \\
\epsilon & \varrho^{*}\left(\left[P u_{0}\right]_{\alpha},\left[Q u_{1}\right]_{\alpha}\right) s\left(\sigma\left(u_{1}, u_{2}\right)\right) .
\end{aligned}
$$


Using Definition 1, we conclude that

$$
\begin{aligned}
Q^{*}\left(\left[P u_{0}\right]_{\alpha},\left[Q u_{1}\right]_{\alpha}\right)\left(\sigma\left(u_{1}, u_{2}\right)\right) & \preceq \zeta \sigma\left(u_{0}, u_{1}\right) \\
& +\frac{\zeta \sigma\left(u_{0},\left[P u_{0}\right]_{\alpha}\right) \sigma\left(u_{1},\left[Q u_{1}\right]_{\alpha}\right)+\eta \sigma\left(u_{1},\left[P u_{0}\right]_{\alpha}\right) \sigma\left(u_{0},\left[Q u_{1}\right]_{\alpha}\right)}{1+\sigma\left(u_{0}, u_{1}\right)} \\
& +\frac{\rho \sigma\left(u_{0},\left[P u_{0}\right]_{\alpha}\right) \sigma\left(u_{0},\left[Q u_{1}\right]_{\alpha}\right)+\delta \sigma\left(u_{1},\left[P u_{0}\right]_{\alpha}\right) \sigma\left(u_{1},\left[Q u_{1}\right]_{\alpha}\right)}{1+\sigma\left(u_{0}, u_{1}\right)}
\end{aligned}
$$

Utilizing the glb property of $P$ and $Q$, we obtain

$$
\begin{aligned}
\varrho^{*}\left(\left[P u_{0}\right]_{\alpha},\left[Q u_{1}\right]_{\alpha}\right)\left(\sigma\left(u_{1}, u_{2}\right)\right) & \preceq \xi \sigma\left(u_{0}, u_{1}\right) \\
& +\frac{\zeta \sigma\left(u_{0}, u_{1}\right) \sigma\left(u_{1}, u_{2}\right)+\eta \sigma\left(u_{1}, u_{1}\right) \sigma\left(u_{0}, u_{2}\right)}{1+\sigma\left(u_{0}, u_{1}\right)} \\
& +\frac{\rho \sigma\left(u_{0}, u_{1}\right) \sigma\left(u_{0}, u_{2}\right)+\delta \sigma\left(u_{1}, u_{1}\right) \sigma\left(u_{1}, u_{2}\right)}{1+\sigma\left(u_{0}, u_{1}\right)}
\end{aligned}
$$

which implies that

$$
\sigma\left(u_{1}, u_{2}\right) \prec \varrho^{*}\left(\left[P u_{0}\right]_{\alpha},\left[Q u_{1}\right]_{\alpha}\right)\left(\sigma\left(u_{1}, u_{2}\right)\right) \preceq \xi \sigma\left(u_{0}, u_{1}\right)+\frac{\zeta \sigma\left(u_{0}, u_{1}\right) \sigma\left(u_{1}, u_{2}\right)}{1+\sigma\left(u_{0}, u_{1}\right)}+\frac{\rho \sigma\left(u_{0}, u_{1}\right) \sigma\left(u_{0}, u_{2}\right)}{1+\sigma\left(u_{0}, u_{1}\right)} .
$$

Thus,

$$
\begin{aligned}
\left|\sigma\left(u_{1}, u_{2}\right)\right| & \leq \xi\left|\sigma\left(u_{0}, u_{1}\right)\right|+\frac{\zeta\left|\sigma\left(u_{0}, u_{1}\right)\right|\left|\sigma\left(u_{1}, u_{2}\right)\right|}{1+\left|\sigma\left(u_{0}, u_{1}\right)\right|}+\frac{\rho\left|\sigma\left(u_{0}, u_{1}\right)\right|\left[\left|\sigma\left(u_{0}, u_{1}\right)\right|+\left|\sigma\left(u_{1}, u_{2}\right)\right|\right]}{1+\left|\sigma\left(u_{0}, u_{1}\right)\right|} \\
& \leq \xi\left|\sigma\left(u_{0}, u_{1}\right)\right|+\zeta\left|\sigma\left(u_{1}, u_{2}\right)\right|+\rho\left|\sigma\left(u_{0}, u_{1}\right)\right|+\rho\left|\sigma\left(u_{1}, u_{2}\right)\right|,
\end{aligned}
$$

which yields

$$
\begin{aligned}
\left|\sigma\left(u_{1}, u_{2}\right)\right| & \leq \frac{\xi+\rho}{1-\zeta-\rho}\left|\sigma\left(u_{0}, u_{1}\right)\right| \\
& =\mathfrak{Z}\left|\sigma\left(u_{0}, u_{1}\right)\right| .
\end{aligned}
$$

From (4), we get

$$
\left|\sigma\left(u_{1}, u_{2}\right)\right| \leq \mathfrak{Z}(1-\mathfrak{Z}) t
$$

Now, consider

$$
\left|\sigma\left(u_{0}, u_{2}\right)\right| \leq \sigma\left|u_{0}, u_{1}\right|+\sigma\left|u_{1}, u_{2}\right| \leq(1-\mathfrak{Z})|t|+\mathfrak{Z}(1-\mathfrak{Z})|t|=(1-\mathfrak{Z})(1+\mathfrak{Z})|t|=\left(1-\mathfrak{Z}^{2}\right)|t| .
$$

Therefore, $u_{2} \in \bar{B}\left(u_{0}, t\right)$. Since $\varrho\left(u_{0}, u_{1}\right) \geq 1$ and $\{P, Q\}$ are coupled $\varrho^{*}$-admissible, $Q^{*}\left(\left[P u_{0}\right]_{\alpha},\left[Q u_{1}\right]_{\alpha}\right) \geq 1$. Following (2), we have

$$
\begin{aligned}
& \xi \sigma\left(u_{1}, u_{2}\right)+\frac{\zeta \sigma\left(u_{1},\left[P u_{1}\right]_{\alpha}\right) \sigma\left(u_{2},\left[Q u_{2}\right]_{\alpha}\right)+\eta \sigma\left(u_{2},\left[P u_{1}\right]_{\alpha}\right) \sigma\left(u_{1},\left[Q u_{2}\right]_{\alpha}\right)}{1+\sigma\left(u_{1}, u_{2}\right)} \\
+ & \frac{\rho \sigma\left(u_{1},\left[P u_{1}\right]_{\alpha}\right) \sigma\left(u_{1},\left[Q u_{2}\right]_{\alpha}\right)+\delta \sigma\left(u_{2},\left[P u_{1}\right]_{\alpha}\right) \sigma\left(u_{2},\left[Q u_{2}\right]_{\alpha}\right)}{1+\sigma\left(u_{1}, u_{2}\right)} \\
\epsilon & \varrho^{*}\left(\left[P u_{1}\right]_{\alpha},\left[Q u_{2}\right]_{\alpha}\right) s\left(\left[P u_{1}\right]_{\alpha},\left[Q u_{2}\right]_{\alpha}\right) .
\end{aligned}
$$


Using Lemma 1 (iii), we obtain

$$
\begin{aligned}
& \zeta \sigma\left(u_{1}, u_{2}\right)+\frac{\zeta \sigma\left(u_{1},\left[P u_{1}\right]_{\alpha}\right) \sigma\left(u_{2},\left[Q u_{2}\right]_{\alpha}\right)+\eta \sigma\left(u_{2},\left[P u_{1}\right]_{\alpha}\right) \sigma\left(u_{1},\left[Q u_{2}\right]_{\alpha}\right)}{1+\sigma\left(u_{1}, u_{2}\right)} \\
+ & \frac{\rho \sigma\left(u_{1},\left[P u_{1}\right]_{\alpha}\right) \sigma\left(u_{1},\left[Q u_{2}\right]_{\alpha}\right)+\delta \sigma\left(u_{2},\left[P u_{1}\right]_{\alpha}\right) \sigma\left(u_{2},\left[Q u_{2}\right]_{\alpha}\right)}{1+\sigma\left(u_{1}, u_{2}\right)} \\
\epsilon & \varrho^{*}\left(\left[P u_{1}\right]_{\alpha},\left[Q u_{2}\right]_{\alpha}\right) s\left(u_{2},\left[Q u_{2}\right]_{\alpha}\right) .
\end{aligned}
$$

By definition, there is some $u_{3} \in\left[Q u_{2}\right]_{\alpha}$ such that

$$
\begin{aligned}
& \xi \sigma\left(u_{1}, u_{2}\right)+\frac{\zeta \sigma\left(u_{1},\left[P u_{1}\right]_{\alpha}\right) \sigma\left(u_{2},\left[Q u_{2}\right]_{\alpha}\right)+\eta \sigma\left(u_{2},\left[P u_{1}\right]_{\alpha}\right) \sigma\left(u_{1},\left[Q u_{2}\right]_{\alpha}\right)}{1+\sigma\left(u_{1}, u_{2}\right)} \\
+ & \frac{\rho \sigma\left(u_{1},\left[P u_{1}\right]_{\alpha}\right) \sigma\left(u_{1},\left[Q u_{2}\right]_{\alpha}\right)+\delta \sigma\left(u_{2},\left[P u_{1}\right]_{\alpha}\right) \sigma\left(u_{2},\left[Q u_{2}\right]_{\alpha}\right)}{1+\sigma\left(u_{1}, u_{2}\right)} \\
\epsilon & Q^{*}\left(\left[P u_{1}\right]_{\alpha},\left[Q u_{2}\right]_{\alpha}\right) s\left(\sigma\left(u_{2}, u_{3}\right)\right) .
\end{aligned}
$$

By Definition 1, we get

$$
\begin{aligned}
\varrho^{*}\left(\left[P u_{1}\right]_{\alpha},\left[Q u_{2}\right]_{\alpha}\right)\left(\sigma\left(u_{2}, u_{3}\right)\right) & \preceq \xi \sigma\left(u_{1}, u_{2}\right) \\
& +\frac{\zeta \sigma\left(u_{1},\left[P u_{1}\right]_{\alpha}\right) \sigma\left(u_{2},\left[Q u_{2}\right]_{\alpha}\right)+\eta \sigma\left(u_{2},\left[P u_{1}\right]_{\alpha}\right) \sigma\left(u_{1},\left[Q u_{2}\right]_{\alpha}\right)}{1+\sigma\left(u_{1}, u_{2}\right)} \\
& +\frac{\rho \sigma\left(u_{1},\left[P u_{1}\right]_{\alpha}\right) \sigma\left(u_{1},\left[Q u_{2}\right]_{\alpha}\right)+\delta \sigma\left(u_{2},\left[P u_{1}\right]_{\alpha}\right) \sigma\left(u_{2},\left[Q u_{2}\right]_{\alpha}\right)}{1+\sigma\left(u_{1}, u_{2}\right)}
\end{aligned}
$$

Utilizing the the glb property of $P$ and $Q$, we conclude that

$$
\begin{aligned}
\varrho^{*}\left(\left[P u_{1}\right]_{\alpha},\left[Q u_{2}\right]_{\alpha}\right)\left(\sigma\left(u_{2}, u_{3}\right)\right) & \preceq \xi \sigma\left(u_{1}, u_{2}\right) \\
& +\frac{\zeta \sigma\left(u_{1}, u_{2}\right) \sigma\left(u_{2}, u_{3}\right)+\eta \sigma\left(u_{2}, u_{2}\right) \sigma\left(u_{1}, u_{3}\right)}{1+\sigma\left(u_{1}, u_{2}\right)} \\
& +\frac{\rho \sigma\left(u_{1}, u_{2}\right) \sigma\left(u_{1}, u_{3}\right)+\delta \sigma\left(u_{2}, u_{2}\right) \sigma\left(u_{2}, u_{3}\right)}{1+\sigma\left(u_{1}, u_{2}\right)}
\end{aligned}
$$

which implies that

$$
\begin{aligned}
\sigma\left(u_{2}, u_{3}\right) \prec Q^{*}\left(\left[P u_{1}\right]_{\alpha},\left[Q u_{2}\right]_{\alpha}\right)\left(\sigma\left(u_{2}, u_{3}\right)\right) & \preceq \xi \sigma\left(u_{1}, u_{2}\right)+\frac{\zeta \sigma\left(u_{1}, u_{2}\right) \sigma\left(u_{2}, u_{3}\right)}{1+\sigma\left(u_{1}, u_{2}\right)} \\
& +\frac{\rho \sigma\left(u_{1}, u_{2}\right)\left[\sigma\left(u_{1}, u_{2}\right)+\sigma\left(u_{2}, u_{3}\right)\right]}{1+\sigma\left(u_{1}, u_{2}\right)} .
\end{aligned}
$$

Hence,

$$
\begin{aligned}
\left|\sigma\left(u_{2}, u_{3}\right)\right| & \leq \xi\left|\sigma\left(u_{1}, u_{2}\right)\right|+\frac{\zeta\left|\sigma\left(u_{1}, u_{2}\right)\right|\left|\sigma\left(u_{2}, u_{3}\right)\right|}{1+\left|\sigma\left(u_{1}, u_{2}\right)\right|}+\frac{\rho\left|\sigma\left(u_{1}, u_{2}\right)\right|\left[\left|\sigma\left(u_{1}, u_{2}\right)\right|+\left|\sigma\left(u_{2}, u_{3}\right)\right|\right]}{1+\left|\sigma\left(u_{1}, u_{2}\right)\right|} \\
& \leq \xi\left|\sigma\left(u_{1}, u_{2}\right)\right|+\zeta\left|\sigma\left(u_{2}, u_{3}\right)\right|+\rho\left|\sigma\left(u_{1}, u_{2}\right)\right|+\rho\left|\sigma\left(u_{2}, u_{3}\right)\right| .
\end{aligned}
$$

Therefore,

$$
\begin{aligned}
\left|\sigma\left(u_{2}, u_{3}\right)\right| & \leq \frac{\xi+\rho}{1-\zeta-\rho}\left|\sigma\left(u_{1}, u_{2}\right)\right| \\
& =\mathfrak{Z}\left|\sigma\left(u_{1}, u_{2}\right)\right|
\end{aligned}
$$

where $\mathfrak{Z}<1$. From (4), we get 


$$
\left|\sigma\left(u_{2}, u_{3}\right)\right| \leq \mathfrak{Z}^{2}(1-\mathfrak{Z}) t
$$

Since

$$
\left|\sigma\left(u_{0}, u_{3}\right)\right| \leq\left|\sigma\left(u_{0}, u_{1}\right)\right|+\sigma\left|u_{1}, u_{2}\right|+\sigma\left|u_{2}, u_{3}\right| \leq(1-\mathfrak{Z})+\mathfrak{Z}\left(1-\mathfrak{Z}|t|+\mathfrak{Z}^{2}(1-\mathfrak{Z})|t|=\left(1-\mathfrak{Z}^{3}\right)|t|,\right.
$$

which show that $u_{3} \in \bar{B}\left(u_{0}, t\right)$. By continuing the process, one can construct a sequence $\left\{u_{p}\right\}$ in $\bar{B}\left(u_{0}, t\right)$ with $p=0,1,2,3, \ldots$

$$
\varrho\left(u_{2 p}, u_{2 p+1}\right) \geq 1 \quad \text { and } \quad \varrho\left(u_{2 p+1} u_{2 p+2}\right) \geq 1
$$

such that

$$
\varrho\left(u_{2 p}, u_{2 p+1}\right) \leq \mathfrak{Z}^{2 p}\left|\sigma\left(u_{0}, u_{1}\right)\right| \quad \text { and } \quad \varrho\left(u_{2 p+1}, u_{2 p+2}\right) \leq \mathfrak{Z}^{2 p+1}\left|\sigma\left(u_{0}, u_{1}\right)\right| \text {, }
$$

where

$$
u_{2 p+1} \in\left[P u_{2 p}\right]_{\alpha} \quad \text { and } \quad u_{2 p} \in\left[Q u_{2 p-1}\right]_{\alpha} .
$$

By induction, we can construct a sequence in $\mathcal{S}$ such that for $p=0,1,2, .$.

$$
\varrho\left(u_{p}, u_{p+1}\right) \geq 1 \quad \text { and } \quad\left|\sigma\left(u_{p}, u_{p+1}\right)\right| \leq \mathfrak{Z}^{p}\left|\sigma\left(u_{0}, u_{1}\right)\right| .
$$

Suppose $q>p$, then, utilizing (5) and triangular inequality, we obtain

$$
\begin{aligned}
\left|\sigma\left(u_{p}, u_{q}\right)\right| & \leq\left|\sigma\left(u_{p}, u_{p+1}\right)\right|+\left|\sigma\left(u_{p+1}, u_{p+2}\right)\right|+\cdots+\left|\sigma\left(u_{q-1}, u_{q}\right)\right| \\
& \leq\left[\mathfrak{Z}^{p}+\mathfrak{Z}^{p+1}+\cdots+\mathfrak{Z}^{q-1}\right]\left|\sigma\left(u_{0}, u_{1}\right)\right| \\
& \leq \frac{\mathfrak{Z}^{p}}{1-\mathfrak{Z}}\left|\sigma\left(u_{0}, u_{1}\right)\right| \rightarrow 0, \quad \text { when } \mathfrak{Z} \rightarrow \infty .
\end{aligned}
$$

This shows that $\left\{u_{p}\right\}$ is a Cauchy sequence in $\bar{B}\left(u_{0}, t\right)$. Since $\mathcal{S}$ is complete and $\bar{B}\left(u_{0}, t\right)$ is a closed subspace of $\mathcal{S}$, therefore, there is $z \in \bar{B}\left(u_{0}, t\right)$ such that $u_{p} \rightarrow z$ when $p \rightarrow \infty$. Finally, we are to show that $z \in[P z]_{\alpha}$ and $z \in[Q z]_{\alpha}$. As $\varrho\left(u_{p}, z\right) \geq 1$ and $\{P, Q\}$ are $\varrho^{*}$-admissible, so $\varrho^{*}\left(\left[P u_{p}\right]_{\alpha},[Q z]_{\alpha}\right) \geq 1$ for all $p$. In the light of Equation (2), we obtain

$$
\begin{aligned}
& \xi \sigma\left(u_{2 p}, z\right)+\frac{\zeta \sigma\left(u_{2 p},\left[P u_{2 p}\right]_{\alpha}\right) \sigma\left(z,[Q z]_{\alpha}\right)+\eta \sigma\left(z,\left[P u_{2 p}\right]_{\alpha}\right) \sigma\left(u_{2 p},[Q z]_{\alpha}\right)}{1+\sigma\left(u_{2 p}, z\right)} \\
+ & +\frac{\rho \sigma\left(u_{2 p},\left[P u_{2 p}\right]_{\alpha}\right) \sigma\left(u_{2 p},[Q z]_{\alpha}\right)+\delta \sigma\left(z,\left[P u_{2 p}\right]_{\alpha}\right) \sigma\left(z,[Q z]_{\alpha}\right)}{1+\sigma\left(u_{2 p}, z\right)} \\
\in & Q^{*}\left(\left[P u_{2 p}\right]_{\alpha},[Q z]_{\alpha}\right) s\left(\left[P u_{2 p}\right]_{\alpha},[Q z]_{\alpha}\right) .
\end{aligned}
$$

Using Lemma 1 (iii), we obtain

$$
\begin{aligned}
& \xi \sigma\left(u_{2 p}, z\right)+\frac{\zeta \sigma\left(u_{2 p},\left[P u_{2 p}\right]_{\alpha}\right) \sigma\left(z,[Q z]_{\alpha}\right)+\eta \sigma\left(z,\left[P u_{2 p}\right]_{\alpha}\right) \sigma\left(u_{2 p},[Q z]_{\alpha}\right)}{1+\sigma\left(u_{2 p}, z\right)} \\
+ & +\frac{\rho \sigma\left(u_{2 p},\left[P u_{2 p}\right]_{\alpha}\right) \sigma\left(u_{2 p},[Q z]_{\alpha}\right)+\delta \sigma\left(z,\left[P u_{2 p}\right]_{\alpha}\right) \sigma\left(z,[Q z]_{\alpha}\right)}{1+\sigma\left(u_{2 p}, z\right)} \\
\in & Q^{*}\left(\left[P u_{2 p}\right]_{\alpha},[Q z]_{\alpha}\right) s\left(u_{2 p+1},[Q z]_{\alpha}\right) .
\end{aligned}
$$


By the definition, there is some $u_{p} \in[Q z]_{\alpha}$, such that

$$
\begin{aligned}
& \xi \sigma\left(u_{2 p}, z\right)+\frac{\zeta \sigma\left(u_{2 p},\left[P u_{2 p}\right]_{\alpha}\right) \sigma\left(z,[Q z]_{\alpha}\right)+\eta \sigma\left(z,\left[P u_{2 p}\right]_{\alpha}\right) \sigma\left(u_{2 p},[Q z]_{\alpha}\right)}{1+\sigma\left(u_{2 p}, z\right)} \\
+ & +\frac{\rho \sigma\left(u_{2 p},\left[P u_{2 p}\right]_{\alpha}\right) \sigma\left(u_{2 p},[Q z]_{\alpha}\right)+\delta \sigma\left(z,\left[P u_{2 p}\right]_{\alpha}\right) \sigma\left(z,[Q z]_{\alpha}\right)}{1+\sigma\left(u_{2 p}, z\right)} \\
\in & Q^{*}\left(\left[P u_{2 p}\right]_{\alpha},[Q z]_{\alpha}\right) s\left(\sigma\left(u_{2 p+1}, u_{p}\right)\right) .
\end{aligned}
$$

By Definition 1, we get

$$
\begin{aligned}
Q^{*}\left(\left[P u_{2 p}\right]_{\alpha},[Q z]_{\alpha}\right)\left(\sigma\left(u_{2 p+1}, u_{p}\right)\right) & \preceq \xi \sigma\left(u_{2 p}, z\right) \\
& +\frac{\zeta \sigma\left(u_{2 p},\left[P u_{2 p}\right]_{\alpha}\right) \sigma\left(z,[Q z]_{\alpha}\right)+\eta \sigma\left(z,\left[P u_{2 p}\right]_{\alpha}\right) \sigma\left(u_{2 p},[Q z]_{\alpha}\right)}{1+\sigma\left(u_{2 p}, z\right)} \\
& +\frac{\rho \sigma\left(u_{2 p},\left[P u_{2 p}\right]_{\alpha}\right) \sigma\left(u_{2 p},[Q z]_{\alpha}\right)+\delta \sigma\left(z,\left[P u_{2 p}\right]_{\alpha}\right) \sigma\left(z,[Q z]_{\alpha}\right)}{1+\sigma\left(u_{2 p}, z\right)} .
\end{aligned}
$$

Utilizing the the glb property of $P$ and $Q$, we obtain

$$
\begin{aligned}
\varrho^{*}\left(\left[P u_{2 p}\right]_{\alpha},[Q z]_{\alpha}\right)\left(\sigma\left(u_{2 p+1}, u_{p}\right)\right) & \preceq \xi \sigma\left(u_{2 p}, z\right) \\
& +\frac{\zeta \sigma\left(u_{2 p}, u_{2 p+1}\right) \sigma\left(z, u_{p}\right)+\eta \sigma\left(z, u_{2 p+1}\right) \sigma\left(u_{2 p}, u_{p}\right)}{1+\sigma\left(u_{2 p}, z\right)} \\
& +\frac{\rho \sigma\left(u_{2 p}, u_{2 p+1}\right) \sigma\left(u_{2 p}, u_{p}\right)+\delta \sigma\left(z, u_{2 p+1}\right) \sigma\left(z, u_{p}\right)}{1+\sigma\left(u_{2 p}, z\right)},
\end{aligned}
$$

which implies that

$$
\begin{aligned}
\sigma\left(u_{2 p+1}, u_{p}\right) \prec \varrho^{*}\left(\left[P u_{2 p}\right]_{\alpha},[Q z]_{\alpha}\right)\left(\sigma\left(u_{2 p+1}, u_{p}\right)\right) & \preceq \xi \sigma\left(u_{2 p}, z\right) \\
& +\frac{\zeta \sigma\left(u_{2 p}, u_{2 p+1}\right) \sigma\left(z, u_{p}\right)+\eta \sigma\left(z, u_{2 p+1}\right) \sigma\left(u_{2 p}, u_{p}\right)}{1+\sigma\left(u_{2 p}, z\right)} \\
& +\frac{\rho \sigma\left(u_{2 p}, u_{2 p+1}\right) \sigma\left(u_{2 p}, u_{p}\right)+\delta \sigma\left(z, u_{2 p+1}\right) \sigma\left(z, u_{p}\right)}{1+\sigma\left(u_{2 p}, z\right)},
\end{aligned}
$$

so we conclude that

$$
\begin{aligned}
\sigma\left(u_{2 p+1}, u_{p}\right) & \preceq \xi \sigma\left(u_{2 p}, z\right)+\frac{\zeta \sigma\left(u_{2 p}, u_{2 p+1}\right) \sigma\left(z, u_{p}\right)+\eta \sigma\left(z, u_{2 p+1}\right) \sigma\left(u_{2 p}, u_{p}\right)}{1+\sigma\left(u_{2 p}, z\right)} \\
& +\frac{\rho \sigma\left(u_{2 p}, u_{2 p+1}\right) \sigma\left(u_{2 p}, u_{p}\right)+\delta \sigma\left(z, u_{2 p+1}\right) \sigma\left(z, u_{p}\right)}{1+\sigma\left(u_{2 p}, z\right)}
\end{aligned}
$$

since

$$
\sigma\left(z, u_{p}\right) \preceq \sigma\left(z, u_{p+1}\right)+\sigma\left(u_{p+1}, u_{p}\right) .
$$

Therefore, following (6), we have

$$
\begin{aligned}
\sigma\left(z, u_{p}\right) & \preceq \sigma\left(z, u_{p+1}\right)+\xi \sigma\left(u_{2 p}, z\right)+\frac{\zeta \sigma\left(u_{2 p}, u_{2 p+1}\right) \sigma\left(z, u_{p}\right)+\eta \sigma\left(z, u_{2 p+1}\right) \sigma\left(u_{2 p}, u_{p}\right)}{1+\sigma\left(u_{2 p}, z\right)} \\
& +\frac{\rho \sigma\left(u_{2 p}, u_{2 p+1}\right) \sigma\left(u_{2 p}, u_{p}\right)+\delta \sigma\left(z, u_{2 p+1}\right) \sigma\left(z, u_{p}\right)}{1+\sigma\left(u_{2 p}, z\right)},
\end{aligned}
$$

which implies that 


$$
\begin{aligned}
\left|\sigma\left(z, u_{p}\right)\right| & \leq\left|\sigma\left(z, u_{p+1}\right)\right|+\xi\left|\sigma\left(u_{2 p}, z\right)\right|+\frac{\zeta\left|\sigma\left(u_{2 p}, u_{2 p+1}\right)\right|\left|\sigma\left(z, u_{p}\right)\right|+\eta\left|\sigma\left(z, u_{2 p+1}\right)\right|\left|\sigma\left(u_{2 p}, u_{p}\right)\right|}{1+\left|\sigma\left(u_{2 p}, z\right)\right|} \\
& +\frac{\rho\left|\sigma\left(u_{2 p}, u_{2 p+1}\right)\right|\left|\sigma\left(u_{2 p}, u_{p}\right)\right|+\delta\left|\sigma\left(z, u_{2 p+1}\right)\right| \sigma\left(z, u_{p}\right) \mid}{1+\left|\sigma\left(u_{2 p}, z\right)\right|}
\end{aligned}
$$

If we take limit as $p \rightarrow \infty$, then we obtain $\left|\sigma\left(z, u_{p}\right)\right| \rightarrow 0$, that is, $u_{p} \rightarrow z$ when $p \rightarrow \infty$. Since $[P z]_{\alpha}$ is closed therefore, $z \in[P z]_{\alpha}$. In the same way, we can obtain that $z \in[Q z]_{\alpha}$. Hence, $P$ and $Q$ have a common fuzzy fixed point.

Theorem 2. Let $(\mathcal{S}, \sigma)$ be complete complex-valued metric space and $P: \mathcal{S} \rightarrow \mathfrak{L}(\mathcal{S})$ be coupled $\varrho^{*}$-admissible mapping such that glb property holds. If $P$ satisfies

$$
\begin{aligned}
& \xi \sigma(u, v)+\frac{\zeta \sigma\left(u,[P u]_{\alpha}\right) \sigma\left(v,[P v]_{\alpha}\right)+\eta \sigma\left(v,[P u]_{\alpha}\right) \sigma\left(u,[P v]_{\alpha}\right)}{1+\sigma(u, v)} \\
+ & \frac{\rho \sigma\left(u,[P u]_{\alpha}\right) \sigma\left(u,[P v]_{\alpha}\right)+\delta \sigma\left(v,[P u]_{\alpha}\right) \sigma\left(v,[P v]_{\alpha}\right)}{1+\sigma(u, v)} \\
\in & \varrho^{*}\left([P u]_{\alpha},[P v]_{\alpha}\right) s\left([P u]_{\alpha},[P v]_{\alpha}\right)
\end{aligned}
$$

for all $v, u \in \bar{B}\left(u_{0}, t\right)$, where $u_{0} \in \mathcal{S}$ and $0 \prec t \in C$, then

$$
(1-\mathfrak{Z}) t \in s\left(u_{0},\left[P u_{0}\right]_{\alpha}\right),
$$

where $\zeta, \eta$ and $\xi$ are nonnegative real numbers such that $\mathfrak{Z}=\frac{\xi+\rho}{1-\zeta-\rho}<1$. Suppose $\varrho\left(u_{0}, u_{1}\right) \geq 1$ for some $u_{1} \in \bar{B}\left(u_{0}, t\right)$ and, for each $v, u \in \mathcal{S}$ associated with some $\alpha \in(0,1]$, there exists $[P u]_{\alpha}$ that is a nonempty closed, bounded subset of $\mathcal{S}$. If $\left\{u_{q}\right\}$ is a sequence in $\bar{B}\left(u_{0}, t\right)$ with $\varrho\left(u_{q}, u_{q+1}\right) \geq 1$ and $u_{q} \rightarrow z$ as $q \rightarrow+\infty$, then $\varrho\left(u_{q}, z\right) \geq 1$ for all $q$. Then, there exists a point $u^{*} \in \bar{B}\left(u_{0}, t\right)$ such that $u^{*} \in\left[P u^{*}\right]_{\alpha} \cap\left[Q u^{*}\right]_{\alpha}$.

Proof. By letting $P=Q$ in Theorem 1, we obtain the above corollary.

Example 1. Let $X_{1}=\{w \in C: \operatorname{Im}(w) \geq 0$ and $\operatorname{Re}(w)=0\}$ and $X_{2}=\{w \in C: \operatorname{Re}(w) \geq 0$ and $\operatorname{Im}(w)=0\}$ and let $X=X_{1} \cup X_{2}$. Consider a metric $d: X \times X \rightarrow C$ as follows:

$$
d\left(w_{1}, w_{2}\right)= \begin{cases}\frac{1}{4}\left|y_{1}-y_{2}\right|+\frac{i}{2}\left|y_{1}-y_{2}\right| & \text { if } w_{1}, w_{2} \in X_{1}, \\ \frac{1}{2}\left|x_{1}-x_{2}\right|+\frac{i}{3}\left|x_{1}-x_{2}\right| & \text { if } w_{1}, w_{2} \in X_{2}, \\ \frac{2}{9}\left|x_{2}+y_{1}\right|+\frac{i}{6}\left|x_{2}+y_{1}\right| & \text { if } w_{1} \in X_{1}, w_{2} \in X_{2}, \\ \frac{1}{3}\left|x_{1}+y_{2}\right|+\frac{2 i}{9}\left|x_{1}+y_{2}\right| & \text { if } w_{1} \in X_{2}, w_{2} \in X_{1},\end{cases}
$$

where $w_{1}=x_{1}+i y_{1}, w_{1}=x_{2}+i y_{2} \in X$. Then, $(X, d)$ is a complex valued metric space. Take $w_{0}=0+\frac{1}{2} i$ and $t=\frac{1}{2}+\frac{1}{3} i$. Then,

$$
\overline{B\left(w_{0}, t\right)}=\left\{w \in X_{1}: 0 \leq \operatorname{Im}(w) \leq 1\right\} \cup\left\{w \in X_{2}: 0 \leq \operatorname{Re}(w) \leq 1\right\}
$$

and

$$
\varrho\left(w_{1}, w_{2}\right)= \begin{cases}1, & \text { if } w_{1}, w_{2} \in \overline{B\left(w_{0}, t\right)} \\ \frac{4}{3}, & \text { otherwise. }\end{cases}
$$


Define $P, Q: X \rightarrow \mathfrak{L}(X)$ by

$$
\begin{gathered}
P(z)(w) \begin{cases}0.5 & \text { if } z=\frac{y}{4}+0 i \in X_{1} \text { with } 0 \leq \operatorname{Im}(w) \leq 1, \operatorname{Re}(w)=0, \\
0.7 & \text { if } z=0+\frac{7 y}{8} i \in X_{1} \text { with } \operatorname{Im}(w)>1, \operatorname{Re}(w)=0, \\
0.4 & \text { if } z=0+\frac{x}{5} i \in X_{2} \text { with } 0 \leq \operatorname{Re}(w) \leq 1, \operatorname{Im}(w)=0, \\
0.2 & \text { if } z=\frac{6 x}{7}+0 i \in X_{2} \text { with } \operatorname{Re}(w)>1, \operatorname{Im}(w)=0,\end{cases} \\
Q(z)(w) \begin{cases}0.5 & \text { if } z=\frac{y}{6}+0 i \in X_{1} \text { with } 0 \leq \operatorname{Im}(w) \leq 1, \operatorname{Re}(w)=0, \\
0.7 & \text { if } z=0+\frac{6 y}{7} i \in X_{1} \text { with } \operatorname{Im}(w)>1, \operatorname{Re}(w)=0, \\
0.4 & \text { if } z=0+\frac{x}{7} i \in X_{2} \text { with } 0 \leq \operatorname{Re}(w) \leq 1, \operatorname{Im}(w)=0, \\
0.2 & \text { if } z=\frac{7 x}{8}+0 i \in X_{2} \text { with } \operatorname{Re}(w)>1, \operatorname{Im}(w)=0 .\end{cases}
\end{gathered}
$$

Then,

$$
\begin{gathered}
{[P w]_{0.7}=\left\{0+\frac{7 y}{8} i\right\}, \quad[P w]_{0.5}=\left\{\frac{y}{4}+0 i, 0+\frac{7 y}{8} i\right\},} \\
{[P w]_{0.4}=\left\{\frac{y}{4}+0 i, 0+\frac{7 y}{8} i, 0+\frac{x}{5} i\right\}, \quad[P w]_{0.2}=\left\{\frac{y}{4}+0 i, 0+\frac{7 y}{8} i, 0+\frac{x}{5} i, \frac{6 x}{7}+0 i\right\},}
\end{gathered}
$$

and

$$
\begin{gathered}
{[Q w]_{0.7}=\left\{0+\frac{6 y}{7} i\right\},[Q w]_{0.5}=\left\{\frac{y}{6}+0 i, 0+\frac{6 y}{7} i\right\},} \\
{[Q w]_{0.4}=\left\{\frac{x}{6}+0 i, 0+\frac{6 y}{7} i, 0+\frac{y}{7} i\right\},[Q w]_{0.2}=\left\{\frac{y}{6}+0 i, \frac{7 x}{8}+0 i, 0+\frac{x}{7} i, 0+\frac{6 y}{7} i\right\},}
\end{gathered}
$$

by a routine calculation, one can verify that the mappings $P$ and $Q$ satisfy the conditions (2) and (3) of Theorem 1 with $\xi=\frac{1}{9}, \zeta=\frac{1}{24}, \eta=\frac{1}{2}, \rho=\frac{1}{36}$ and $\delta=\frac{1}{37}$. Hence, $P$ and $Q$ are contractions on $\overline{B\left(w_{0}, r\right)}$.

It is interesting to notice that $P$ and $Q$ are not contractions on the whole space $X$ for $w_{1}=w_{2}=0+\frac{4}{3} i \notin$ $B\left(w_{0}, r\right)$ and for $\alpha=0.7$, as

$$
\begin{aligned}
& \varrho^{*}\left(\left[P w_{1}\right]_{\alpha},\left[Q w_{2}\right]_{\alpha}\right) \sigma\left(\left[P w_{1}\right]_{\alpha},\left[Q w_{2}\right]_{\alpha}\right) \\
= & \frac{4}{3}\left(\frac{1}{168}+\frac{1}{84} i\right) \succ \frac{7,235}{1,342,656}+\frac{5,788}{1,342,656} i \\
= & \xi \sigma\left(w_{1}, w_{2}\right)+\frac{\zeta \sigma\left(w_{1},\left[P w_{1}\right]_{\alpha}\right) \sigma\left(w_{2},\left[Q w_{2}\right]_{\alpha}\right)+\eta \sigma\left(w_{2},\left[P w_{1}\right]_{\alpha}\right) \sigma\left(w_{1},\left[Q u_{2}\right]_{\alpha}\right)}{1+\sigma\left(w_{1}, w_{2}\right)} \\
+ & \frac{\rho \sigma\left(w_{1},\left[P w_{1}\right]_{\alpha}\right) \sigma\left(w_{1},\left[Q w_{2}\right]_{\alpha}\right)+\delta \sigma\left(w_{2},\left[P w_{1}\right]_{\alpha}\right) \sigma\left(w_{2},\left[Q u_{2}\right]_{\alpha}\right)}{1+\sigma\left(w_{1}, w_{2}\right)} .
\end{aligned}
$$

Therefore,

$$
\xi \sigma\left(w_{1}, w_{2}\right)+\frac{\zeta \sigma\left(w_{1},\left[P w_{1}\right]_{\alpha}\right) \sigma\left(w_{2},\left[Q w_{2}\right]_{\alpha}\right)+\eta \sigma\left(w_{2},\left[P w_{1}\right]_{\alpha}\right) \sigma\left(w_{1},\left[Q u_{2}\right]_{\alpha}\right)}{1+\sigma\left(w_{1}, w_{2}\right)}+
$$

$\frac{\rho \sigma\left(w_{1},\left[P w_{1}\right]_{\alpha}\right) \sigma\left(w_{1},\left[Q w_{2}\right]_{\alpha}\right)+\delta \sigma\left(w_{2},\left[P w_{1}\right]_{\alpha}\right) \sigma\left(w_{2},\left[Q u_{2}\right]_{\alpha}\right)}{1+\sigma\left(w_{1}, w_{2}\right)} \notin Q^{*}\left(\left[P w_{1}\right]_{\alpha},\left[Q w_{2}\right]_{\alpha}\right) s\left(\left[P w_{1}\right]_{\alpha},\left[Q w_{2}\right]_{\alpha}\right)$. 
Theorem 3. Let $(\mathcal{S}, \sigma)$ be complete complex valued metric space and $A_{1}, A_{2}: \mathcal{S} \rightarrow \mathcal{C B}(\mathcal{S})$ be coupled $\varrho^{*}$-admissible mappings such that glb property holds. If $A_{1}$ and $A_{2}$ satisfy

$$
\begin{aligned}
& \xi \sigma(u, v)+\frac{\zeta \sigma\left(u, A_{1} u\right) \sigma\left(v, A_{2} v\right)+\eta \sigma\left(v, A_{1} u\right) \sigma\left(u, A_{2} v\right)}{1+\sigma(u, v)} \\
+ & \frac{\rho \sigma\left(u, A_{1} u\right) \sigma\left(u, A_{2} v\right)+\delta \sigma\left(v, A_{1} u\right) \sigma\left(v, A_{2} v\right)}{1+\sigma(u, v)} \\
\epsilon & \varrho^{*}\left(A_{1} u, A_{2} v\right) s\left(A_{1} u, A_{2} v\right)
\end{aligned}
$$

for all $v, u \in \bar{B}\left(u_{0}, t\right)$, where $u_{0} \in \mathcal{S}$ and $0 \prec t \in \mathcal{C}$, then

$$
(1-\mathfrak{Z}) t \in s\left(u_{0}, A_{1} u_{0}\right)
$$

where $\zeta, \eta$ and $\xi$ are nonnegative real numbers such that $\mathfrak{Z}=\frac{\xi+\rho}{1-\zeta-\rho}<1$. Suppose $\varrho\left(u_{0}, u_{1}\right) \geq 1$ for some $u_{1} \in \bar{B}\left(u_{0}, t\right)$. If $\left\{u_{q}\right\}$ is a sequence in $\bar{B}\left(u_{0}, t\right)$ with $\varrho\left(u_{q}, u_{q+1}\right) \geq 1$ and $u_{q} \rightarrow z$ as $q \rightarrow+\infty$, then $\varrho\left(u_{q}, z\right) \geq 1$ for all $q$. Then, there exists a point $u^{*} \in \bar{B}\left(u_{0}, t\right)$ such that $u^{*} \in A_{1} u^{*} \cap A_{2} u^{*}$.

Proof. Let $P, Q: \mathcal{S} \rightarrow \mathfrak{S}(\mathcal{S}), i=1,2$ be fuzzy mappings defined as

$$
\begin{aligned}
& P(u)= \begin{cases}\propto, & \text { if } u \in A_{1} u, \\
0, & \text { if } u \notin A_{1} u,\end{cases} \\
& Q(u)= \begin{cases}\propto, & \text { if } u \in A_{2} u, \\
0, & \text { if } u \notin A_{2} u .\end{cases}
\end{aligned}
$$

Then, for any $\alpha \in(0,1],[P u]_{\alpha}=A_{1} u$ and $[P u]_{\alpha}=A_{2} u$.

Since for every $u, v \in \mathcal{S}, s\left([P u]_{\alpha},[Q v]_{\alpha}\right)=s\left(A_{1} u, A_{2} v\right)$, therefore, one can apply Theorem 1 to obtain some $u \in \mathcal{S}$ such that $u \in A_{1}(u) \cap A_{2}(u)$.

Corollary 1. Let $(\mathcal{S}, \sigma)$ be complete complex valued metric space and $A: \mathcal{S} \rightarrow \mathcal{C B}(\mathcal{S})$ be coupled $\varrho^{*}$-admissible mapping such that glb property holds. If A satisfies

$$
\begin{aligned}
& \xi \sigma(u, v)+\frac{\zeta \sigma(u, A u) \sigma(v, A v)+\eta \sigma(v, A u) \sigma(u, A v)}{1+\sigma(u, v)} \\
+ & \frac{\rho \sigma(u, A u) \sigma(u, A v)+\delta \sigma(v, A u) \sigma(v, A v)}{1+\sigma(u, v)} \\
\epsilon & \varrho^{*}(A u, A v) s(A u, A v)
\end{aligned}
$$

for all $v, u \in \bar{B}\left(u_{0}, t\right)$, where $u_{0} \in \mathcal{S}$ and $0 \prec t \in \mathcal{C}$, then

$$
(1-\mathfrak{Z}) t \in s\left(u_{0}, A u_{0}\right)
$$

where $\zeta, \eta$ and $\xi$ are nonnegative real numbers such that $\mathfrak{Z}=\frac{\xi+\rho}{1-\zeta-\rho}<1$. Suppose $\varrho\left(u_{0}, u_{1}\right) \geq 1$ for some $u_{1} \in \bar{B}\left(u_{0}, t\right)$. If $\left\{u_{q}\right\}$ is a sequence in $\bar{B}\left(u_{0}, t\right)$ with $\varrho\left(u_{q}, u_{q+1}\right) \geq 1$ and $u_{q} \rightarrow z$ as $q \rightarrow+\infty$, then $\varrho\left(u_{q}, z\right) \geq 1$ for all $q$. Then, there exists a point $u^{*} \in \bar{B}\left(u_{0}, t\right)$ such that $u^{*} \in A u^{*} \cap A u^{*}$.

Proof. Proof is immediate by setting $A_{1}=A_{2}=A$ in Corollary 3. 
Remark 2. (1) In Theorem 1, if condition (3) is replaced by

$$
(1-\mathfrak{Z}) t \in s\left(u_{0}, Q u_{0}\right)
$$

then the result remains the same.

(2) By setting $\rho=\delta=0$ in Theorem 3, we get Theorem 2.9 of [34].

(3) By setting $\rho=\delta=0$ in Corollary 1, we get Theorem 2.12 of [34].

(4) By setting $\eta=\rho=\delta=0$ in Theorem 3, we get Corollary 2.11 of [34].

(5) By setting $\zeta=\eta=\rho=\delta=0$ in Theorem 3, we get Corollary 2.13 of [34].

\section{Application}

Theorem 4. Let $(\mathcal{S}, \sigma)$ be a complete complex valued metric space and let $\mathcal{U}$ be an open subset of $\mathcal{S}$. Let $F$ : $[0,1] \times \overline{\mathcal{U}} \rightarrow \mathcal{C B}(\mathcal{S})$ be multivalued mapping with the glb property. Suppose that there exist $\eta^{o} \in \mathcal{S}$ and $0 \prec \epsilon \in C$ such that

(a) $\eta \notin[F(r, \eta)]$ for all $\eta \in \partial \mathcal{U}$ and $r \in[0,1]$;

(b) $F(r,):. \overline{\mathcal{U}} \rightarrow \mathcal{C B}(\mathcal{S})$ is a multivalued mapping satisfying

$$
\begin{aligned}
& \xi \sigma\left(\eta, \eta^{\prime}\right)+\frac{\zeta \sigma(\eta, F(r, \eta)) \sigma\left(\eta^{\prime}, F\left(r^{\prime}, \eta^{\prime}\right)\right)+\varrho \sigma\left(\eta^{\prime}, F(r, \eta)\right) \sigma\left(\eta, F\left(r^{\prime}, \eta^{\prime}\right)\right)}{1+\sigma\left(\eta, \eta^{\prime}\right)} \\
+ & \frac{\rho \sigma(\eta, F(r, \eta)) \sigma\left(\eta, F\left(r^{\prime}, \eta^{\prime}\right)\right)+\delta \sigma\left(\eta^{\prime}, F(r, \eta)\right) \sigma\left(\eta^{\prime}, F\left(r^{\prime}, \eta^{\prime}\right)\right)}{1+\sigma\left(\eta, \eta^{\prime}\right)} \\
\in & s\left(F(r, \eta), F\left(r^{\prime}, \eta^{\prime}\right)\right),
\end{aligned}
$$

$$
(1-\mathfrak{Z}) \epsilon \in s\left(\eta^{o}, F\left(r^{o}, \eta^{o}\right)\right),
$$

where $\zeta, \eta$ and $\xi$ are nonnegative real numbers such that $\mathfrak{Z}=\frac{\xi+\rho}{1-\zeta-\rho}<1$.

(c) there exists a continuous increasing function $\Phi:(0,1] \rightarrow K \cup\{0\}$ such that

$$
\Phi(s)-\Phi(r) \in s\left(F(s, \eta), F\left(r, \eta^{\prime}\right)\right), \quad \Phi(s) \in \Phi(r)
$$

for all $s, r \in[0,1]$ and $\eta \in \overline{\mathcal{U}}$ where $K=\{w \in C: 0 \prec w\}$.

Then, $F(0,$.$) has a fixed point if and only if F(1,$.$) has a fixed point.$

Proof. Let $F(0,$.$) have a fixed point w$, so $w \in F(0, w)$.

With the help of assumption $a$ ), we can define the following set:

$$
\mathcal{X}=\{(r, \eta) \in[0,1] \times \mathcal{U}: \eta \in F(r, \eta)\} .
$$

Clearly, $\mathcal{X} \neq \phi$. One can define partial ordering $\preceq$ as below

$$
\begin{gathered}
(r, \eta) \preceq\left(s, \eta^{\prime}\right) \text { if } r \leq s, \\
\sigma\left(\eta, \eta^{\prime}\right) \preceq \frac{2}{1-\mathfrak{Z}}(\Phi(s)-\Phi(r)) .
\end{gathered}
$$

Assume that $M$ is a totaly ordered subset of $\mathcal{X}$ and $r^{o}=\sup \{r:(r, \eta) \in M\}$. Consider $\left\{\left(r_{k}, \eta_{k}\right)_{k \geq 0}\right\}$ to be a sequence in $M$ such that $\left(r_{k}, \eta_{k}\right) \preceq\left(r_{k+1}, \eta_{k+1}\right)$ and $r_{k} \rightarrow r^{o}$ as $k \rightarrow \infty$. Then, for any $k \geq 1$ with $l>k$, we obtain

$$
\sigma\left(\eta_{l}, \eta_{k}\right) \preceq \frac{2}{1-\mathfrak{Z}}\left(\Phi\left(r_{l}\right)-\Phi\left(r_{k}\right)\right) \rightarrow 0
$$


as $k, l \rightarrow \infty$, which yields that $\left\{\eta_{k}\right\}$ is a Cauchy sequence. Since $(\mathcal{S}, \sigma)$ is a complete complex valued metric space, there exists $\eta^{o} \in \mathcal{S}$ such that $\eta_{k} \rightarrow \eta^{o}$. From $(a)$, let $k_{0} \in N$ such that, for all $k \geq k_{0}$,

$$
\begin{aligned}
& \xi \sigma\left(\eta_{k}, \eta^{o}\right)+\frac{\zeta \sigma\left(\eta_{k}, F\left(r_{k}, \eta_{k}\right)\right) \sigma\left(\eta^{o}, F\left(r^{o}, \eta^{o}\right)\right)+\varrho \sigma\left(\eta^{o}, F\left(r_{k}, \eta_{k}\right)\right) \sigma\left(\eta_{k}, F\left(r^{o}, \eta^{o}\right)\right)}{1+\sigma\left(\eta_{k}, \eta^{o}\right)} \\
+ & \frac{\rho \sigma\left(\eta_{k}, F\left(r_{k}, \eta_{k}\right)\right) \sigma\left(\eta_{k}, F\left(r^{o}, \eta^{o}\right)\right)+\delta \sigma\left(\eta^{o}, F\left(r_{k}, \eta_{k}\right)\right) \sigma\left(\eta^{o}, F\left(r^{o}, \eta^{o}\right)\right)}{1+\sigma\left(\eta_{k}, \eta^{o}\right)} \\
\in & s\left(F\left(r_{k}, \eta_{k}\right), F\left(r^{o}, \eta^{o}\right)\right), \\
& \xi \sigma\left(\eta_{k}, \eta^{o}\right)+\frac{\zeta \sigma\left(\eta_{k}, F\left(r_{k}, \eta_{k}\right)\right) \sigma\left(\eta^{o}, F\left(r^{o}, \eta^{o}\right)\right)+\rho \sigma\left(\eta^{o}, F\left(r_{k}, \eta_{k}\right)\right) \sigma\left(\eta_{k}, F\left(r^{o}, \eta^{o}\right)\right)}{1+\sigma\left(\eta_{k}, \eta^{o}\right)} \\
+ & \frac{\rho \sigma\left(\eta_{k}, F\left(r_{k}, \eta_{k}\right)\right) \sigma\left(\eta_{k}, F\left(r^{o}, \eta^{o}\right)\right)+\delta \sigma\left(\eta^{o}, F\left(r_{k}, \eta_{k}\right)\right) \sigma\left(\eta^{o}, F\left(r^{o}, \eta^{o}\right)\right)}{1+\sigma\left(\eta_{k}, \eta^{o}\right)} \\
\in & s\left(\eta_{k}, F\left(r^{o}, \eta^{o}\right)\right) .
\end{aligned}
$$

Since $\eta_{k} \in F\left(r_{k}, \eta_{k}\right)$, there exists $\eta_{n} \in F\left(r^{o}, \eta^{o}\right)$ such that

$$
\begin{aligned}
\sigma\left(\eta_{k}, \eta_{n}\right) & \preceq \xi \sigma\left(\eta_{k}, \eta^{o}\right)+\frac{\zeta \sigma\left(\eta_{k}, F\left(r_{k}, \eta_{k}\right)\right) \sigma\left(\eta^{o}, F\left(r^{o}, \eta^{o}\right)\right)+\varrho \sigma\left(\eta^{o}, F\left(r_{k}, \eta_{k}\right)\right) \sigma\left(\eta_{k}, F\left(r^{o}, \eta^{o}\right)\right)}{1+\sigma\left(\eta_{k}, \eta^{o}\right)} \\
& +\frac{\rho \sigma\left(\eta_{k}, F\left(r_{k}, \eta_{k}\right)\right) \sigma\left(\eta_{k}, F\left(r^{o}, \eta^{o}\right)\right)+\delta \sigma\left(\eta^{o}, F\left(r_{k}, \eta_{k}\right)\right) \sigma\left(\eta^{o}, F\left(r^{o}, \eta^{o}\right)\right)}{1+\sigma\left(\eta_{k}, \eta^{o}\right)} .
\end{aligned}
$$

Utilizing glb property of $F$, we obtain

$$
\begin{aligned}
\sigma\left(\eta_{k}, \eta_{n}\right) & \preceq \xi \sigma\left(\eta_{k}, \eta^{o}\right)+\frac{\varrho \sigma\left(\eta^{o}, \eta_{k}\right) \sigma\left(\eta_{k}, \eta_{n}\right)}{1+\sigma\left(\eta_{k}, \eta^{o}\right)} \\
& +\frac{\delta \sigma\left(\eta^{o}, \eta_{k}\right) \sigma\left(\eta^{o}, \eta_{n}\right)}{1+\sigma\left(\eta_{k}, \eta^{o}\right)}
\end{aligned}
$$

which implies that

$$
\begin{aligned}
\left|\sigma\left(\eta_{k}, \eta_{n}\right)\right| & \leq \xi\left|\sigma\left(\eta_{k}, \eta^{o}\right)\right|+\varrho\left|\frac{\sigma\left(\eta^{o}, \eta_{k}\right)}{1+\sigma\left(\eta_{k}, \eta^{o}\right)}\right| \sigma\left(\eta_{k}, \eta_{n}\right) \mid \\
& +\delta\left|\frac{\sigma\left(\eta^{o}, \eta_{k}\right)}{1+\sigma\left(\eta_{k}, \eta^{o}\right)}\right|\left|\sigma\left(\eta^{o}, \eta_{n}\right)\right|,
\end{aligned}
$$

which yields

$$
\begin{aligned}
\left|\sigma\left(\eta_{k}, \eta_{n}\right)\right| & \leq \xi\left|\sigma\left(\eta_{k}, \eta^{o}\right)\right|+\rho\left|\sigma\left(\eta_{k}, \eta_{n}\right)\right|+\delta\left|\sigma\left(\eta^{o}, \eta_{n}\right)\right|, \\
& \leq \xi\left|\sigma\left(\eta_{k}, \eta^{o}\right)\right|+\rho\left|\sigma\left(\eta_{k}, \eta_{n}\right)\right|+\delta\left[\left|\sigma\left(\eta^{o}, \eta_{k}\right)\right|+\left|\sigma\left(\eta_{k}, \eta_{n}\right)\right|\right], \\
& \leq \frac{\xi+\delta}{1-\rho-\delta}\left|\sigma\left(\eta^{o}, \eta_{k}\right)\right| .
\end{aligned}
$$

Consider

$$
\begin{aligned}
\left|\sigma\left(\eta^{o}, \eta_{n}\right)\right| & \leq\left|\sigma\left(\eta^{o}, \eta_{k}\right)\right|+\left|\sigma\left(\eta_{k}, \eta_{n}\right)\right| \\
& \leq\left|\sigma\left(\eta^{o}, \eta_{k}\right)\right|+\frac{\xi+\delta}{1-\rho-\delta}\left|\sigma\left(\eta^{o}, \eta_{k}\right)\right| \rightarrow 0
\end{aligned}
$$

for all $k>k_{0}$. Hence, $\eta_{n} \rightarrow \eta^{o} \in F\left(r^{o}, \eta^{o}\right)$ and therefore $\eta^{o} \in \mathcal{U}$ implies that $\left(r^{o}, \eta^{o}\right) \in \mathcal{X}$. Thus, $(r, \eta) \preceq$ $\left(r^{o}, \eta^{o}\right)$ for all $(r, \eta) \in M$; this shows that $\left(r^{o}, \eta^{o}\right)$ is an upper bound of $M$. Therefore, by utilizing 
Zorn's Lemma, we obtain that $\left(r^{o}, \eta^{o}\right)$ is the maximal element of $\mathcal{X}$. Now, we are going to show that $r^{0}=1$. Suppose, on the contrary, that $r^{0} \leq 1$, let $0 \prec \epsilon \in C$ and $r^{o} \leq r$ with

$$
\bar{B}\left(\eta^{o}, \epsilon\right) \subset \mathcal{U} \text { where } \epsilon=\frac{2}{1-\mathfrak{Z}}\left(\Phi(r)-\Phi\left(r^{o}\right)\right) .
$$

In the light of the condition $c$ ), we get

$$
\begin{gathered}
\left(\Phi(r)-\Phi\left(r^{o}\right)\right) \in s\left(F(r, \eta), F\left(r^{o}, \eta^{o}\right)\right) \\
\left(\Phi(r)-\Phi\left(r^{o}\right)\right) \in s\left(F(r, \eta), \eta^{o}\right) \text { for all } \eta^{o} \in F\left(r^{o}, \eta^{o}\right) .
\end{gathered}
$$

Therefore, there exists some $\eta \in F(r, \eta)$, such that

$$
\left(\Phi(r)-\Phi\left(r^{o}\right)\right) \in s\left(\sigma\left(\eta, \eta^{o}\right)\right),
$$

which implies that

$$
\sigma\left(\eta, \eta^{o}\right) \preceq\left(\Phi(r)-\Phi\left(r^{o}\right)\right) \preceq \frac{(1-\mathfrak{Z}) \epsilon}{2} \prec(1-\mathfrak{Z}) \epsilon,
$$

so that

$$
\left|\sigma\left(\eta, \eta^{o}\right)\right| \leq(1-\mathfrak{Z})|\epsilon|
$$

Using the condition $b$, we obtained that the mapping $F(r,):. \bar{B}\left(\eta^{o}, \epsilon\right) \rightarrow \mathcal{C B}(\mathcal{S})$ enjoys all the conditions of Corollary 1 . Thus, there exists $\eta \in \bar{B}\left(\eta^{o}, \epsilon\right)$ such that $\eta \in F(r, \eta)$; therefore, $(\eta, r) \in \mathcal{X}$. As

$$
\sigma\left(\eta, \eta^{o}\right) \prec \epsilon=\frac{2}{1-\mathfrak{Z}}\left(\Phi(r)-\Phi\left(r^{o}\right)\right)
$$

this yields that $\left(r^{o}, \eta^{o}\right) \preceq(r, \eta)$, which is a contradiction; hence, $r=1$. Thus, $F(., 1)$ has a common fixed point. Conversely, by following the same techniques, we can prove that, if $F(1,$.$) has a fixed$ point, then $F(0,$.$) has a fixed point.$

\section{Conclusions}

We have successfully derived common fixed point results for fuzzy mappings by using the concept of coupled $\alpha^{*}$-admissible mapping in complex-valued metric spaces. We have generalized many results in the existing literature by providing the application of our main result to multivalued mappings and a homotopy result.

Author Contributions: All authors contribute equally to the writing of this manuscript. All authors reads and improve the final version.

Funding: This project was supported by the Theoretical and Computational Science (TaCS) Center under the Computational and Applied Science for Smart Innovation Cluster (CLASSIC), Faculty of Science, KMUTT.

Acknowledgments: The authors wish to thank the editor and anonymous referees for their comments and suggestions, which helped to improve this paper.

Conflicts of Interest: The authors declare no conflict of interest.

\section{References}

1. Banach, S. Sur les opérations dans les ensembles abstraits et leurs applications aux équations intégrales. Fund. Math. 1922, 3, 133-181. [CrossRef]

2. Balasubramaniam, P.; Muralisankar, S. Existence and uniqueness of fuzzy solution for the nonlinear fuzzy integrodifferential equations. Appl. Math. Lett. 2001, 4, 455-462. [CrossRef]

3. Ćirić, B.L. A generalization of Banach contraction principal. Proc. Am. Math. Soci. 1974, 2, 267-273. [CrossRef]

4. Nieto, J.J. The Cauchy problem for continuous fuzzy differential equations. Fuzzy Sets Syst. 1999, 102, 259-262. [CrossRef] 
5. Shatanawi, W.; Rajić, V.Ć.; Radenovic, S.; Al-Ravashedeh, A. Mizoguchi-Takahashi-type theorem in tvs cone metric spaces. Fixed Point Theory. Appl. 2012, 106. [CrossRef]

6. Seikkala, S. On the fuzzy initial value problem. Fuzzy Sets Syst. 1987, 24, 319-330. [CrossRef]

7. Song, S.; Guo, L.; Feng, C. Global existence of solutions to fuzzy differential equations. Fuzzy Sets Syst. 2000, 115, 371-376. [CrossRef]

8. Nadler, B.S., Jr. Multivalued contraction mappings. Pac. J. Math. 1969, 30, 475-488. [CrossRef]

9. Azam, A.; Ahmad, J.; Kumam, P. Common fixed point theorems for multivalued mappings in complex valued metric spaces. J. Inequal. Appl. 2013, 2013, 578. [CrossRef]

10. Kaleva, O. Fuzzy differential equations. Fuzzy Sets Sys. 1987, 24, 301-317. [CrossRef]

11. Heilpern, S. Fuzzy fixed point theorems. J. Math. Anal. 1981, 83, 566-569. [CrossRef]

12. Azam, A. Fuzzy fixed points of fuzzy mappings via a rational inequality. Hacet. J. Math. Stat. 2011, 40, 421-431.

13. Humaira; Sarwar, M.; Kishore, G.N.V. Fuzzy fixed point results for contractive mapping with applications. Complexity 2018, 2018, 5303815. [CrossRef]

14. Humaira; Sarwar, M.; Tunç, C. Fuzzy fixed point results via rational type contractions involving control functions in complex-valued metric spaces. Appl. Math. Inf. Sci. 2018, 4, 861-875. [CrossRef]

15. Arora, S.C.; Sharma, V. Fixed points for fuzzy mappings. Fuzzy Sets Syst. 2000, 110, 127-130. [CrossRef]

16. Azam, A.; Beg, I. Common fuzzy fixed point for fuzzy mappings. Fixed Point Theory Appl. 2013, 14. [CrossRef]

17. Bose, R.K.; Sahani, D. Fuzzy mapping and fixed point theorems. Fuzzy Sets Sys. 1987, 21, 53-58. [CrossRef]

18. Estruch, V.D.; Vidal, A. A note on fixed point for fuzzy mappings. Rend Istit. Math. Univ. Trieste 2001, $32,39-45$.

19. Green, A.; Pastor, J. A fixed point theorem for fuzzy contraction mapping. Rend. Istit. Math. Univ. Trieste 1999, XXX, 103-109.

20. Kutbi, M.A.; Ahmad, J.; Azam, A.; Hussain, N. On fuzzy fixed points for fuzzy maps with generalized weak property. J. Appl. Math. 2014, 2014, 549504. [CrossRef]

21. Turkolgue, D.; Rhodes, B.E. A fixed fuzzy point for fuzzy mapping in complete metric space. Math. Commun. 2005, 10, 115-121.

22. Dass, B.K.; Gupta, S. An extension of Banach contraction principle through rational expression. Indian. J. Pure Appl. Math. 1975, 6, 1455-1458.

23. Azam, A.; Fisher, B.; Khan, M. Common fixed point theorems in complex valued metric spaces. Numer. Funct. Anal. Opti. 2011, 32, 243-253. [CrossRef]

24. Sintunavarat, W.; Kumam, P. Generalized common fixed point theorems in complex valued metric spaces and applications. J. Inequal. Appl. 2012, 84. [CrossRef]

25. Sintunavarat, W.; Cho, J.Y.; Kumam, P. Urysohn integral equations approach by common fixed points in complex valued metric spaces. Adv. Differ. Equ. 2013, 49. [CrossRef]

26. Ahmad, J.; Klin-Eam, C.; Azam, A. Common fixed points for multivalued mappings in complex valued metric spaces with applications. Abstr. Appl. Anal. 2013, 2013, 854965. [CrossRef]

27. Klin-eam, C.; Suanoom, C. Some common fixed point theorems for generalized contractive type mappings on complex valued metric spaces. Abstr. Appl. Anal. 2013, 2013, 604215. [CrossRef]

28. Rouzkard, F.; Imdad, M. Some common fixed point theorems on complex valued metric spaces. Comput. Math. Appl. 2012, 64, 1866-1874. [CrossRef]

29. Sitthikul, K.; Saejung, S. Some fixed point theorems in complex valued metric space. Fixed Point Theory Appl. 2012, 189. [CrossRef]

30. Kutbi, M.A.; Ahmad, J.; Azam, A.; Al-Rawashdeh, A.S. Generalized common fixed point results via greatest lower bound property. J. Appl. Math. 2014, 2014, 265865. [CrossRef]

31. Samet, B.; Vetro, C.; Vetro, P. Fixed point theorems for $\alpha-\psi$-contractive type mappings. Nonlinear Anal. 2012, 75, 2154-2165. [CrossRef]

32. Hassanzadeasl, J. Common fixed point theorems for $\alpha-\psi$-contractive type mappings. Int. J. Anal. 2013, 2013, 1-7. [CrossRef] 
33. Kutbi, M.A.; Ahmad, J.; Azam, A. On fixed points of $\alpha-\psi$-contractive multivalued mappings in cone metric spaces. Abstr. Appl. Anal. 2013, 2013, 313782. [CrossRef]

34. Shatanawi, W.; Noorani, M.S.M.D.; Ahmad, J.; Alsamir, H.; Kutbi, M.A. Some common fixed points of multivalued mappings on complex-valued metric spaces with homotopy result. J. Nonlinear Sci. Appl. 2017, 10, 3381-3396. [CrossRef]

(C) 2019 by the authors. Licensee MDPI, Basel, Switzerland. This article is an open access article distributed under the terms and conditions of the Creative Commons Attribution (CC BY) license (http://creativecommons.org/licenses/by/4.0/). 